\title{
Alternativen als Programm
}

\author{
Plädoyer für einen Perspektivenwechsel \\ in der Technikfolgenabschätzung
}

\author{
Paulina Dobroć, Institut für Technikfolgenabschätzung und Systemanalyse (ITAS), Karlsruher Institut für Technologie (KIT), \\ Karlstr.11, 76133 Karlsruhe (paulina.dobroc@kit.edu), (1) orcid.org/0000-0003-4537-8405 \\ Bettina-Johanna Krings, Institut für Technikfolgenabschätzung und Systemanalyse (ITAS/KIT) (bettina-johanna.krings@kit.edu), \\ (1) orcid.org/0000-0002-4253-0136 \\ Christoph Schneider, Friedrich Schiedel-Lehrstuhl für Wissenschaftssoziologie, Technische Universität München \\ (schneider.christoph@tum.de), (1) orcid.org/0000-0003-1516-158X \\ Nele Wulf, Institut für Technikfolgenabschätzung und Systemanalyse (ITAS/KIT) (nele.wulf@kit.edu)
}

Der vorliegende Artikel soll einen Beitrag zu theoretischen Überlegungen einer Technikfolgenabschätzung (TA) der Alternativen leisten. Ausgehend von der Annahme, dass TA seit ihrer Entstehung den Anspruch auf die Entwicklung von (technischen) Alternativen erhoben hat, wird hier der Versuch unternommen, die Rolle der TA im Rahmen aktueller Innovationsprozesse zu reflektieren und (neu) zu definieren. Dies scheint vor allem im Hinblick auf aktuelle gesellschaftliche Herausforderungen notwendig. Zu diesem Zweck wird eine Grundannahme Lothar Hacks über das Verhältnis von Gesellschaft und Technik herangezogen. Seine Annahme besagt, dass die kontinuierliche Überführung von sozialen Problemen und Leitbildern in enge technische Lösungsansätze in hochindustrialisierten Ländern dominant und Teil der aktuellen gesellschaftlichen Probleme ist. Diese These wird im vorliegenden Artikel im Hinblick auf einen Perspektivenwechsel als Ausgangspunkt eines zukünftigen Forschungsprogramms der TA fruchtbar gemacht. Hierbei bildet das Denken in Alternativen von gesellschaftlichen Zukünften den zentralen Fokus.

\section{A program of alternatives}

Pledge for a shift in perspective in technology assessment

This article provides theoretical arguments for a technology assessment (TA) of alternatives that critically engages in innovation and transformation processes. We draw on an observation by Lothar Hack who stated that a major problem of industrial societies is the dominant translation of complex societal problems into narrow technical solutions. Here we have to come up with alternatives to open up space for other options; it also requires a fundamental shift in perspective that may guide the research program of a TA of alternatives. Based on this, we reflect on the role of TA and its expectations in the light of a vastly changed innovation landscape.

This is an article distributed under the terms of the Creative Commons Attribution License CCBY 4.0 (https://creativecommons.org/licenses/by/4.0/)

https://doi.org/10.14512/tatup.27.1.28

Submitted: 17.10.2017. Peer reviewed. Accepted: 29.01.2018
Keywords: technology assessment, technological progress, alternatives, methodological challenges

\section{Einleitung}

Das Fachportal Technikfolgenabschätzung „openTA“ definiert das Ziel der Technikfolgenabschätzung als das „Aufbrechen scheinbarer Sachzwänge zugunsten von Entscheidungsalternativen unter Einbeziehung sozialer bzw. nichttechnischer Optionen“ (openTA 2017). Diese Selbstbeschreibung, die das Aufzeigen von Handlungsoptionen und deren Bewertung betont, ist nicht nur für aktuelle Anforderungen an die TA zentral. Sie stellt ein wichtiges Moment der historischen Entstehungsgeschichte der TA dar. Angesichts signifikanter globaler Herausforderungen, die bisher weitgehend durch technisch ausgerichtete Lösungsprogramme bewältigt werden sollen, wie beispielsweise der globale Klimawandel, scheint es jedoch notwendig - so die Annahme - wieder Räume für das Denken und Handeln in Alternativen zu erwirken. High-Tech-Innovationsprogramme zur Bewältigung solcher Herausforderungen nicht als naturgegebene Notwendigkeit - also als alternativlos - anzunehmen, ist der Ausgangspunkt unserer Argumentation. Genau weil technischen Innovationen eine so prominente Stellung als Veränderungskraft zugeschrieben wird, sollte TA auch andere Formen der Zukunftsgestaltung in den Blick rücken und so Dialoge über Alternativen mit ermöglichen. Statt sich einfach neuen Technologien zuzuwenden, wie es die Forschungsförderer oft verlangen, sollte eine TA der Alternativen einen Schritt zurücktreten und fragen, welches die sozialen und ethischen Prämissen des Handelns sind, bevor sie sich technischen Möglichkeiten zuwendet. Dieses Vorgehen impliziert ein fundamentales „Denken in Alternativen“ über spezifische Technisierungsprogramme hinaus, was gerade vor dem Hintergrund von poli- 
tisch motivierten Debatten um ,Alternativlosigkeit‘ angezeigt scheint.

Mit einem Rückgriff auf die Entstehungs- und Entwicklungsgeschichte der TA, auf unser Verständnis von Alternative als Programm (Teil 2) sowie auf die konzeptionellen Alternativen von Lothar Hack im Hinblick auf das dominante Verhältnis von Gesellschaft und Technik (Teil 3), diskutieren wir im Folgenden die mögliche Entwicklung der TA. TA könnte und sollte ihre (kritische) Distanz zu technischen Innovationen wiederentdecken und gleichzeitig den Anspruch erheben, eine engagierte Mitgestalterin gesellschaftlicher Zukünfte zu sein. Dies erfordert von der TA einen Perspektivenwechsel insofern, als sie nicht mehr men die Proponenten der TA zunächst eine kritische Außenposition ein (Grunwald 2010).

Seither haben sich jedoch Innovationsprozesse und -systeme deutlich geändert und Teile der TA rückten aus einer ehemals distanzierten Position in die aktive Gestaltung von Innovationsprozessen hinein. Dies hat das Selbstverständnis von TA fundamental verändert und wurde auch kritisch reflektiert (Bechmann 2007).

Seit den 1990er-Jahren entwickelte sich mit dem Aufstieg der Gen- und Nanotechnologien das Programm der ELSA-Forschung (Ethical Legal and Social Aspects), welches den Schwerpunkt der Technikbewertung auf ethische, rechtliche und soziale

\section{Technikfolgenabschätzung ist aus einer ehemals distanzierten Position in die aktive Gestaltung von Innovations- prozessen hineingerückt.}

nach der Integration neuer Technologien in bestehende Arrangements fragt, sondern neben der Bewertung von Veränderungsprozessen durch technische Innovationen auch Alternativen zum Status quo eröffnet, initiiert und moderiert. Das Beispiel der Digitalisierung und der FabLabs illustriert dieses Anliegen. Abschließend (Teil 4) wird eine theoretische Konzeptionierung einer kritisch-engagierten TA und ihrer Funktionen im Innovationsgeschehen vorgeschlagen und anhand folgender Fragen profiliert: Wie könnte sich eine TA positionieren, sodass die Entwicklung von Alternativen als Programm (wieder) ins Zentrum ihrer Arbeit rückt? Was würde dies für die TA bedeuten (Teil 5)?

\section{TA und ihre Folgen}

Die „klassische“ TA hat sich seit ihrer Entstehung in den 1970er-Jahren selbst als distanzierte und oftmals dezidiert kritische Beobachterin technischer Entwicklungen und deren Folgen verstanden. TA wurde zunächst im gesellschaftlichen Feld der Politik institutionalisiert. Wissenschaftliche Expertise wurde durch die Politik - eingeholt, um die Folgen und Nebenfolgen von soziotechnischen Phänomenen abzuschätzen, sie zu analysieren und zu bewerten. Diese parlamentarische TA sollte die Handlungs- und Entscheidungsfähigkeit der Parlamente erhöhen und damit zur Demokratisierung des wissenschaftlich-technischen Fortschritts beitragen, ohne allerdings das Dogma der werturteilsfreien Forschung anzutasten. Als expertenbasierte und, neutrale ' Beobachtungsinstanz verstand sie sich selbst als Alternative zu den durch Wirtschaftsinteressen geprägten Technisierungsprojekten der Industrie und erhob den Anspruch, der Politik beratend zur Seite zu stehen. Die Alternative zu technokratischen Sachzwängen damals schien klar: Ausgehend von der Zielvorstellung ,sozialverträglicher' Technikentwicklung nah-
Implikationen legt. Entwicklungen in der Forschung sollten nun auch durch geistes- und sozialwissenschaftliche sowie juristische Expertise aktiv begleitet werden (Rip 2014). Schließlich bringt das Aufkommen von Responsible Research and Innovation (RRI) in den letzten Jahren neue Veränderungen für die TA. RRI zielt auf die umfassende Umgestaltung von Innovationsprozessen, insbesondere durch die Inklusion unterschiedlichster Akteure in Innovationsprozesse sowie deren Ausrichtung auf gesellschaftlich akzeptierte Ziele und Bedarfe (Schomberg 2013). Die Verantwortung für die Gestaltung von Innovationen wird somit idealiter auf viele Stakeholder verteilt. Neben die seit langem dominante Zielsetzung, technische Innovationen als vermarktbare Produkte zu ermöglichen (Pfotenhauer und Juhl 2017), tritt jetzt auch die Veränderung von Innovationsprozessen in ihrer Logik wie in ihren Abläufen neu hinzu.

Der historische Wandel der Positionierung von TA in dieser Landschaft ist markant: Aus einer Ex-post-Bewertung wurde zunehmend eine Ex-ante-Bewertung technologischer Innovation, die dem Anspruch der (Mit-)Gestaltung den der kritischen Bewertung mehr und mehr opferte. Tritt TA als Mitgestalterin technischer Entwicklungen auf, dann kann zu Recht bezweifelt werden, ob sie noch eine distanzierte und kritische Perspektive auf Technik als Erkenntnisgegenstand einzunehmen in der Lage ist (Dolata und Werle 2007). Sie konfrontierte sich durchaus mit dieser (neuen) Position und betonte den eigenen ,produktiven“ und „kritischen“ Beitrag im Rahmen dieser Prozesse (Grunwald 1999, 2003). Gleichwohl kann TA nicht ausblenden, dass der durch technische Konzepte geprägte Innovationsraum die Rahmenbedingungen und Möglichkeitsräume der TA erheblich strukturiert. Dies ist auch der Fall, wenn sie mit Szenariotechniken unterschiedliche Zukunftsoptionen darlegen möchte.

Alternativen zu diesen, auf technische Innovationen und Vermarktungslogik verengten Möglichkeitsräumen sollten - so das 
Anliegen des vorliegenden Artikels - wieder ein wichtiger Teil des Forschungsprogramms der TA werden. Im Folgenden wird auf der Basis von theoretischen Prämissen des Industriesoziologen Lothar Hack verdeutlicht, wie eine kritische Reflektion technischer Innovationen erfolgen könnte und warum eine Neubestimmung der Integration von Technologien in die gesellschaftliche, stets soziotechnische Praxis von großer Bedeutung ist. Abschließend soll die Arbeit an und mit Alternativen erläutert werden.

\section{Alternativen zu dominanten Innovationsperspektiven}

Lothar Hack (1994) formulierte im Rahmen seiner Studien zum informationstechnischen Wandel in Unternehmen einen Zusammenhang, den er als grundlegend für eine theoretische Präzisierung von TA betrachtete und den wir im Rahmen der Reflexionen zu gesellschaftlichen Alternativen fruchtbar machen. Angelehnt an die Erkenntnisse der damaligen Debatte der Wissenschafts- und Technikforschung folgte er der These, dass dort eine wichtige „Neubestimmung des Verhältnisses von ,Technik“ und ,Gesellschaft"“ (Hack 1994, S. 295) vorgenommen würde (Knorr-Cetina 1983; Bijker et al. 1987). Die „Konstruktion der Grenze selbst [würde] zum Problem“ (Hack 1994, S. 295), womit bisherige Gewissheiten im Hinblick auf das Verhältnis Gesellschaft und Technik aufgekündigt würden. Bisher seien das Verhältnis von Gesellschaft und Technik als zwei selbstständige Bereiche beschrieben worden. Jedoch könne deren Verhältnis sehr wohl als ein ,nahtloses Gewebe von technischen, ökonomischen, kulturellen, sozialen und politischen Faktoren, deren respektive Abgrenzungen voneinander erst im Vollzug der Technologieentwicklungen ausgehandelt und verbindlich definiert werden“ (ebd.), beschrieben werden. lungen selbst“" (ebd.). Diese Gemengelage präge das Verhältnis von Gesellschaft und Technik konstitutiv und gestalte so in besonderem Maße auch Zukunftsentwürfe. Diese Beobachtung lässt sich an aktuellen Entwicklungen von neuen technischen Konzepten wie Industrie 4.0 bestätigen, deren Ausrichtung sich an ökonomischen Parametern orientiert, die weiter die soziale Gestaltung industrieller Zukünfte bestimmt (Hirsch-Kreinsen et al. 2015).

Hack stellt im Anschluss an seine Beobachtungen die relevante Frage, wie und warum gesellschaftliche Probleme in „technische, bzw. technologisch bearbeitbare überführt [werden]“ (Hack 1994, S. 295). Warum, so könnte die Frage umgekehrt lauten, bildet in erster Linie der Möglichkeitsraum des Technischen die Blaupause für zukünftige gesellschaftliche Entwicklungen? Die Debatte um die technologisch induzierte Arbeitslosigkeit im Anschluss an Industrie 4.0 weist genau auf diesen Mangel hin. In Anlehnung an Hack (Hack und Hack 1985, 1998; Hack 1988) lautet unsere These, dass die Aufgabe von TA angesichts eines „nahtlosen Gewebes“ von Technik und Gesellschaft gerade darin bestehen sollte, soziale, politische und kulturelle Integrität wiederherzustellen.

Diese Diagnose hat, nach unserer Ansicht nichts von ihrer Aktualität eingebüßt. Die Relevanz der Technikwissenschaften im Hinblick auf normative Zukunftsentwürfe ist eher noch gestiegen. Technisch ausgerichtete Zukunftsvisionen bilden die Grundlage vieler Innovationsanstrengungen, genießen in öffentlichen Debatten hohe Aufmerksamkeit und prägen machtvoll die gesellschaftliche Auseinandersetzung mit Zukunft (Lösch et al. 2016; Grunwald 2016; Nordmann 2016). An Hack anschließend liegen die damit verbundenen Probleme zum einen in der perspektivischen Verengung auf neue Technologien als alleiniger Lösungsoption trotz gesellschaftlicher Komplexität und zum anderen in der diskursiven Stabilisierung kapitalgetriebener Innovationsprozesse, welche kaum demokratisch ablaufen.

\section{Warum bildet in erster Linie der Möglichkeitsraum des Technischen die Blaupause für zukünftige gesellschaftliche Entwicklungen?}

Allerdings - und hier vertritt Hack einen kapitalismuskritischen Anspruch - entwickle sich diese Neubestimmung nicht offen und unbestimmt, sondern verlaufe gemäß den Prämissen der Erhaltung kapitalistischer Ökonomien. Dies bedeute eine ,enge[n] Kopplung von Technologien mit wissenschaftlichem Wissen einerseits und ökonomischen Bewertungen andererseits“ (ebd., S. 296). Kennzeichnend für diese neuere Entwicklung seien hierbei ,die Formen der ,strukturellen Ökonomisierung ' technischer und technologischer Entwicklungen, durch die soziale, kulturelle und politische Bewertungen als ökonomische Gewichtungen Eingang finden in die technologischen Entwick-
Vor dem Hintergrund der dargelegten Überlegungen scheint es uns notwendig, diese Prozesse nicht nur kritisch zu hinterfragen und zu analysieren, sondern etwas darüber hinaus anzubieten: TA sollte es sich zur Aufgabe machen, das Bewusstsein der Kontingenz unseres Denkens und Handelns wach zu halten und diskursive Schließungsprozesse kritisch zu begleiten. Als Schließung wird hier die selektive Durchsetzung einzelner Weltdeutungen verstanden. Zwar sind diese notwendig, um überhaupt handlungsfähig zu sein, aber aus einem demokratischen Verständnis heraus bedarf dies nach unserem Dafürhalten der Aushandlungsprozesse über Alternativen. Diese dürfen sich nicht 
auf ein hegemoniales „Weiter so!“ begrenzen, das sich höchstens in einem „Wie?“ ergeht. Die Frage kann daher nicht lauten, welche andere Technik angemessen wäre, um unsere Probleme anzugehen.

TA könnte und sollte den Raum eröffnen, um (a) hegemoniale Deutungen sichtbar zu machen und ggf. zurückzuweisen und (b) Optionen von technischen Innovationen von der anderen Seite her zu formulieren - nämlich: Wie kann sozialen, kulturellen und politischen Veränderungen mehr Aufmerksamkeit, Spielraum und Legitimation innerhalb soziotechnischer Innovationsprozesse zukommen? Denken in Alternativen heißt vor dem Hintergrund dieser Überlegungen, dass TA nach unserer Überzeugung daran mitwirken sollte, die Frage „Wie wollen wir jetzt und zukünftig leben?" ins Zentrum der Aushandlungen um Innovationsprozesse zu stellen. Sie in allen möglichen Kontexten als notwendiges Element einer Erörterung zu etablieren und selbst zu stellen, sollte die zentrale Aufgabe einer TA der Alternativen sein. Ihre Beantwortung kann und darf, um der Voraus-
TA der Alternativen in diesem Sinne ist dann auch eine Absage an letztgültige und auf Dauer gestellte Lösungen. Zwar sind Schließungsprozesse notwendig für gestaltendes Handeln. Wie wir allerdings gezeigt haben, operiert TA derzeit in einem Feld, in dem diese trotz aller Innovationsdynamik dominieren.

\section{Alternative Möglichkeitsräume: Digitalisierung und FabLabs}

Digitalisierung ist zu einem der führenden politischen Narrative aktueller Transformationsprozesse geworden. So schreibt beispielsweise die Bundesregierung der Bundesrepublik Deutschland in ihren Grundsätzen zur Digitalpolitik: „Der digitale Wandel ist zu einer der zentralen Gestaltungsaufgaben für Wirtschaft, Wissenschaft, Gesellschaft und Politik geworden“ (Deutsche Bundesregierung 2018). Viele gesellschaftliche Zukunftsentwürfe stützen sich derzeit auf Visionen digitaler Technologien,

\section{Denken in Alternativen geht mit einem Öffnungs- prozess einher, der mit der Frage „Wie wollen wir leben?“ einsetzt.}

setzung demokratisch zu sein zu entsprechen, immer nur temporär erfolgen. Kontingenz muss prinzipiell denkbar und ein alternatives Handeln möglich sein.

Denken in Alternativen geht mit einem Öffnungsprozess einher, der mit der Frage „Wie wollen wir leben?“ einsetzt. Die Arbeit mit Alternativen gestaltet sich mehrdimensional. Zunächst sind Alternativen als Konkretisierungen gefragt und nicht als ein reines Beharren auf abstrakter Offenheit. Alternativen sind nicht gegeben, sondern sie müssen erzeugt, erarbeitet und eingeleitet werden. Daher sind sie auch kontingent: Sie werden zu einem bestimmten Zeitpunkt, in einer bestimmten Gesellschaft, von bestimmten Akteuren vertreten. Um sich diese Kontingenz stets vor Augen zu halten, bedarf eine TA der Alternativen eines reflexiven Vorgehens. Ein solches Vorgehen rekurriert auf die etymologische Herleitung des Begriffes "Alternative“ aus dem Lateinischen Verb alternare: ,abwechseln; bald dieses, bald jenes erwägen“. Dies beschreibt den Prozess des Alternativenfindens, aus dem heraus Entwicklung und Innovation entstehen. Statt schlicht den Rahmensetzungen dominanter Innovationsprogramme zu folgen, könnte eine TA der Alternativen über das aktive Stellen der Frage „Wie wollen wir leben?“ die Öffnung und die Erzeugung von Alternativen anregen.

Außer der Begleitung des Prozesses zum Generieren von Alternativen bedeutet die Arbeit an Alternativen auch, ein Bewusstsein über die Vergänglichkeit der konkret untersuchten Alternative zu entwickeln. In der Arbeit an einer Alternative sollte immer wieder nach anderen Alternativen gefragt werden. Eine ohne jedoch den gesellschaftlichen Fortschritt qualitativ zu benennen. Wie hier Räume entstehen können, die Alternativen zu einer Hightech-Strategie hervorbringen, wird am Beispiel der FabLabs vorgeführt (Schneider 2017).

Diese mittlerweile mehr als 1.000, meist kleineren und gemeinschaftlich getragenen, Organisationen haben in den letzten Jahren dazu beigetragen, einen alternativen Pfad für die gesellschaftliche Auseinandersetzung mit digitalen Produktionstechnologien zu ermöglichen, jenseits der industriellen Nutzung. Das Konzept entstand am Massachusetts Institute of Technology (MIT) aus dem starken technikwissenschaftlichen Glauben an die Möglichkeiten neuer Technologien. Laien sollten digitale Fabrikationstechnologien wie 3-D-Drucker, Plotter oder Laser Cutter zunächst durch die Universität zugänglich gemacht werden. Seit 2002 entstanden so unter Schirmherrschaft des MIT einige Dutzend FabLabs.

Dies änderte sich durch Bestrebungen, vom MIT unabhängige FabLabs aufzubauen, was 2010 mit dem ersten gemeinschaftsgetragenen FabLab gelang. Neu waren hieran zwei Dinge: 1) Die Organisationsform eines durch seine Mitglieder getragenen FabLabs. Technik sollte gemeinsam genutzt und Wissen zum individuellen Empowerment geteilt werden. 2) Die Möglichkeiten zur Organisation und Nutzung waren durch die seit 2004 stark gewachsene Szene der Open Source Technikentwicklungsprojekte und deren kollaborativ erstellte Baupläne für 3-D-Drucker und andere Maschinen stark gewachsen. Hierdurch entstanden große Wissensgemeingüter im Internet, von 
denen auch lokal organisierte FabLab Gemeinschaften profitierten.

FabLabs wurden so zu gesellschaftlichen Laboratorien, in denen nicht nur neue Technik, sondern alternative Ökonomien und Organisationsmodelle vorgestellt, ausprobiert und etabliert werden können. Sie sind zu wichtigen Organisationen geworden, die einen dezentralen, auf Individuen, kostengünstige Produktion sowie das Teilen von Wissen ausgerichteten Pfad der digitalen Fabrikationstechnologie mit ermöglicht und stabilisiert haben. Hierin können die neuen Technologien nicht nur ,in-
Ein wichtiger Aspekt dieser Aufgaben ist das Bewusstseins für Kontingenz und die kritische Begleitung der diskursiven und institutionellen Schließungsprozesse. Schließungsprozesse sind als kontextualisierte Entscheidungsprozesse wichtig und notwendig, um institutionell überhaupt handlungsfähig zu sein. Wie wir allerdings im Anschluss an Lothar Hack gezeigt haben, finden diese Schließungen typischerweise als Verengungen auf technische Produkte und industrielle Logiken statt. Eine TA der Alternativen muss daher den mutigen Schritt vollziehen, nicht mit diesen Schließungen zu beginnen, sondern sie aufzubrechen.

\section{Eine Technikfolgenabschätzung der Alternativen stellt die Frage:} Wie können wir gesellschaftliche Problemlagen in bearbeitbare Lösungsstrategien überführen?

dustriell" genutzt werden, sondern mit einer Orientierung auf Gemeingüter hin. Es handelt sich hierbei um einen Öffnungsund Veränderungsprozess, der über die Jahre neue Möglichkeitsräume im Denken und Handeln geschaffen hat und so Alternativen sichtbar werden lässt.

\section{Alternativen (mit-)gestalten - neue Herausforderungen für die TA}

Öffnen sich alternative Möglichkeitsräume, so kann dies, wie im obigen Beispiel skizziert, alternative soziale Praktiken, aber auch Technikentwicklungen ermöglichen. Dies kann zweifellos auch ohne TA geschehen. Gleichwohl könnte TA zukünftig die Rolle der (Mit-)Gestalterin einnehmen, indem sie ihren ursprünglichen Anspruch der Demokratisierung von Technik aktiv verfolgt und weiterentwickelt. Sie würde hier im Dia$\log$ mit beteiligten Akteuren soziale und technische Möglichkeitsräume auskundschaften, in denen bewusst nach Alternativen zu den herrschenden Technikvisionen gefragt wird. Dies würde in zweierlei Hinsicht besondere Herausforderungen an TA stellen: Zum Ersten wären wissenschaftliche Analysen um das Verhältnis von Gesellschaft und technischen Innovationen nach wie vor wichtiger Bestandteil der Methoden von TA; zum Zweiten würde der Praxisbezug, d. h. das Öffnen gesellschaftlicher Alternativräume, ein wichtiges methodisches Element darstellen, das als Arbeit-am-Prozess beschrieben werden kann. Immerhin kann TA in diesem Bereich schon auf weitreichende Expertise aufbauen (Grunwald 2010) und darüber hinaus auf Inspirationen aus der transformativen Forschung (Schneidewind 2015) sowie auf Arbeiten zu dialogischen WissenschaftsÖffentlichkeits-Verhältnissen (Burawoy 2005) zurückgreifen. Neben Analysen sind hierfür engagierte Wissenschaftskommunikation, Aktionsforschung oder realexperimentelle Ansätze gefragt.
Alternativen (plural) als Programm geht bewusst auf den Prozess der Generierung von Alternativen ein und stellt eine dialogische, fragmentarische, prozessuale, kontextorientierte und offene Art der Forschung dar (vgl. Böschen et al. 2016). Das Werden und die Etablierung der Alternativen, nicht ihr So-Sein, sollte hierbei hervorgehoben werden. Eine TA der Alternativen zeichnet sich durch einen Gestaltungscharakter aus. Ihren Auftrag sucht sie darin, öffentlich vermittelte Alternativen zu generieren und zu entwickeln.

Im Programm einer TA der Alternativen geht es im Wesentlichen um die Frage, wie wir gesellschaftliche Problemlagen in bearbeitbare Lösungsstrategien überführen können. Und ganz im Sinne Hacks geht es dabei insbesondere darum, Denk- und Handlungsräume jenseits der dominanten und industriell verengten Innovationsprozesse zu finden, die zu sehr die Auseinandersetzung mit Zukunft durch TA bestimmen. Technische Ansätze stellen hierbei wichtige Parameter dar, die Orientierung erfolgt jedoch nicht mehr über die Mobilisierung technischer Optionen, sondern über die Frage ,Wie wir jetzt und zukünftig leben wollen?“ Diese Neuorientierung bedeutet einen Perspektivenwechsel, der das praktizierte Verhältnis von Gesellschaft und Technik grundlegend verändern kann und soll. Nichts weniger als die Mitgestaltung daran könnte der Auftrag einer TA der Alternativen sein.

\footnotetext{
Literatur

Bechmann, Gotthard (2007): Die Beschreibung der Zukunft als Chance oder Risiko? TA zwischen Innovation und Prävention. In: TATuP - Zeitschrift für Technikfolgenabschätzung in Theorie und Praxis 16 (1), S.34-44.

Bijker, Wiebe E.; Hughes, Thomas P.; Pinch, Trevor J. (1987): The social construction of technological systems. New directions in the sociology and history of technology. Cambridge, MA: MIT Press.

Böschen, Stefan; Dusseldorp, Marc; Krings, Bettina-Johanna (2016): Transformation des Wissenssystems. In: Torsten Meireis (Hg.): Nachhaltigkeit (Jahrbuch Sozialer Protestantismus, Band 9). Gütersloh: Gütersloher Verlagshaus, S. 183-212.
} 
Burawoy, Michael (2005): For public sociology. In: American Sociological Review 70, S. 4-28.

Deutsche Bundesregierung (2018): Grundsätze unserer Digitalpolitik. Online verfügbar unter https://www.digitale-agenda.de/Webs/DA/DE/Grundsaetze/ Grundsaetze_Digitalpolitik/grundsaetze-digitalpolitik_node.html, zuletzt geprüft am 13.01.2018.

Dolata, Ulrich; Werle, Raymund (Hg.) (2007): Gesellschaft und die Macht der Technik. Sozioökonomischer und institutioneller Wandel durch Technisierung. Frankfurt a. M.: Campus.

Grunwald, Armin (1999): Technology assessment or ethics of technology? Reflections on technology development between social sciences and philosophy. In: Ethical perspectives: a quarterly review, S. 10-182.

Grunwald, Armin (2010): Technikfolgenabschätzung. Eine Einführung. Berlin: edition sigma.

Grunwald, Armin (2016): The hermeneutic side of responsible research and innovation. Hoboken, NJ: John Wiley \& Sons.

Hack, Lothar (1988): Zur Vollendung der Tatsachen. Die Rolle von Wissenschaft und Technologie in der „dritten Phase der Industriellen Revolution“. Frankfurt a. M.: Fischer.

Hack, Lothar (1994): Reichweiten. Theoriegeleitete Technikforschung und TA. In: Gotthard Bechmann und Thomas Petermann (Hg.): Interdisziplinäre Technikforschung. Genese, Folgen, Diskurs. Frankfurt a. M.: Campus.

Hack, Lothar; Hack, Irmgard (1985): Die Wirklichkeit, die Wissen schafft. Zum wechselseitigen Begründungsverhältnis von „Verwissenschaftlichung der Industrie" und "Industrialisierung der Wissenschaft". Frankfurt a. M.: Campus.

Hack, Lothar; Hack, Irmgard (1998): Technologietransfer und Wissenstransformation. Zur Globalisierung der Forschungsorganisation von Siemens. Münster: Westfälisches Dampfboot.

Hirsch-Kreinsen, Hartmut; Ittermann, Peter; Niehaus, Jonathan (Hg.) (2015): Digitalisierung industrieller Arbeit. Die Vision Industrie 4.0 und ihre sozialen Herausforderungen. Baden-Baden: Nomos.

Knorr-Cetina, Karin D. (1983): The ethnographic study of scientific work. Towards a constructivist interpretation of science. In: Karin D. Knorr-Cetina (Hg.):

Science observed. Perspectives on the social study of science. London: Sage, S. 115-140.

Lösch, Andreas; Böhle, Knud; Coenen, Christopher; Dobroć, Paulina; Ferrari, Arianna; Heil, Reinhard et al. (2016): Technikfolgenabschätzung von soziotechnischen Zukünften. ITZ-Diskussionspapier Nr. 03/2016. Karlsruhe: KIT. DOI: 10.5445/IR/1000062676.

Nordmann, Alfred (2016): Enhancing machine nature. In: J. Benjamin Hurlbut und Hava Tirosh-Samuelson (Hg.): Perfecting human futures. Wiesbaden: Springer Fachmedien, S. 195-214.

openTA - Fachportal Technikfolgenabschätzung (2017): Online verfügbar unter https://www.openta.net/was-ist-ta, zuletzt geprüft am 13.01.2018.

Pfotenhauer, Sebastian M.; Juhl, Joakim (2017): Innovation and the political state. Beyond the myth of technologies and markets. In: Critical studies of innovation. Alternative approaches to the pro-innovation bias. Cheltenham, UK: Edward Elgar, S. 68-93.

Rip, Arie (2014): The past and future of RRI. In: Life sciences, society and policy 10, S. 17. DOI: 10.1186/s40504-014-0017-4.

Schneider, Christoph (2017): Transforming TechKnowledgies. The case of open digital fabrication. Dissertation. Technische Universität München, München.
Schneidewind, Uwe (2015): Transformative Wissenschaft. Motor für gute Wissenschaft und lebendige Demokratie. In: GAIA - Ecological Perspectives for Science and Society 24 (2), S. 88-91.

Schomberg, René von (2013): A vision of responsible research and innovation. In: Richard Owen, John Bessant und Maggy Heintz (Hg.): Responsible innovation. Managing the responsible emergence of science and innovation in society. Hoboken, NJ: John Wiley \& Sons, S. 51-74.

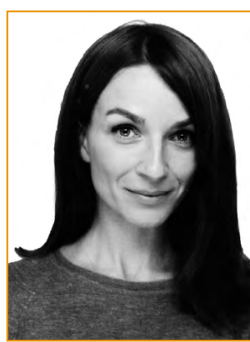

\section{PAULINA DOBROĆ}

ist Doktorandin am Institut für Technikfolgenabschätzung (ITAS) und am Institut für Germanistik des KIT und Stipendiatin der Hans-Böckler-Stiftung. Sie beschäftigt sich mit Visionen-Analyse, Semiotik und Diskursanalyse. In ihrer Dissertation arbeitet sie zum Open-Source-Diskurs.

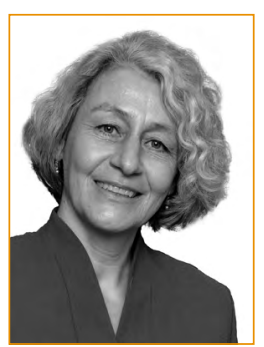

\section{DR. BETTINA-JOHANNA KRINGS}

ist Sozialwissenschaftlerin am Institut für Technikfolgenabschätzung (ITAS) des Karlsruher Instituts für Technologie (KIT) wo sie zu den thematischen Schwerpunkten Technologien und Auswirkungen auf Arbeitsstrukturen, Methoden der Technikfolgenabschätzung und Theorien der Modernisierung von Gesellschaften arbeitet. Am ITAS ist sie Ko-Leiterin des Forschungsbereichs "Wissensgesellschaft und Wissenspolitik".

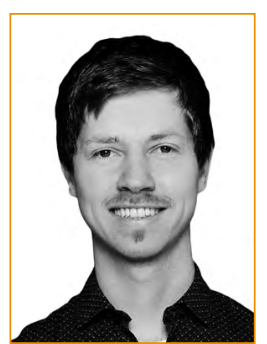

\section{DR. CHRISTOPH SCHNEIDER}

ist Postdoc am Lehrstuhl für Wissenschaftssoziologie der TU München. In Forschung und Lehre arbeitet er zur Demokratisierung von Technologie und öffentlicher Soziologie.

\section{NELE WULE}

ist Soziologin und arbeitet als wissenschaftliche Mitarbeiterin am Institut für Technikfolgenabschätzung und Systemanalyse (ITAS) des Karlsruher Instituts für Technologie (KIT). Als Doktorandin im Projekt ENTRIA beschäftigt sie sich mit der nuklearen Endlagerung in Schweden aus Perspektive der Soziologie des Nichtwissens. 